\title{
Besonderheiten bei der Notfallversorgung von pädiatrischen Patienten
}

Thomas Rielage, Fabian Biederbick

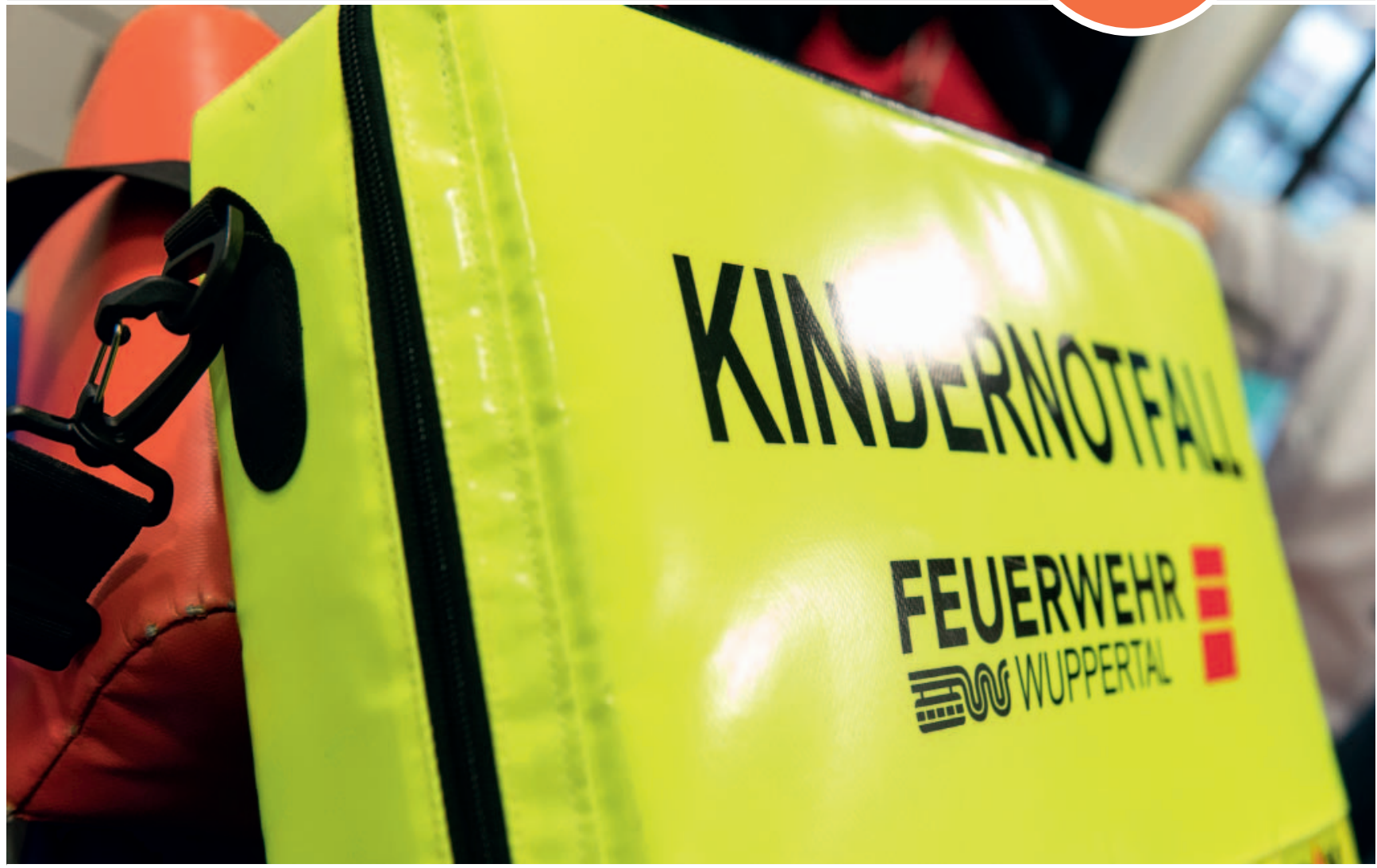

Quelle: KH Krauskopf

Kindernotfälle stellen Mitarbeiter im Rettungsdienst vor besondere Herausforderungen. Zu den psychischen Belastungsfaktoren gehören neben einem relativ geringen Aufkommen von Kindernotfällen von etwa 4 \% aller Notfälle [1] weitere psychologische, physiologische, aber auch anatomische Besonderheiten, die im Folgenden beleuchtet werden. Es wird auf typische Fallstricke eingegangen.

\section{ABKÜRZUNGEN \\ AAP American Academy of Pediatrics \\ AHA American Heart Association \\ HWS Halswirbelsäule \\ NEF Notarzteinsatzfahrzeug \\ PALS $^{\circledR} \quad$ Paediatric Advanced Life Support \\ PAT $^{\circledR} \quad$ Paediatric Assessment Triangle \\ RTW Rettungswagen \\ $\mathrm{S}_{\mathrm{p}} \mathrm{O}_{2} \quad$ pulsoxymetrisch gemessene Sauerstoffsättigung}

\section{FALLBEISPIEL}

Mit dem Einsatzstichwort „allergische Reaktion mit Luftnot" werden an einem Sonntagnachmittag ein RTW und ein NEF in ein Wohngebiet alarmiert. Nach Eintreffen des RTW erfolgt umgehend eine Rückmeldung an die Leitstelle und die durch längere Anfahrt verzögert eintreffende NEF-Besatzung: Es handelt sich um ein 5-jähriges Mädchen mit ausgeprägten Schwellungen im Mund-Rachen-Raum, bei Bewusstsein, jedoch dyspnoisch. 
Bis zum Eintreffen des NEFs können folgende Vitalparameter erhoben werden: $\mathrm{S}_{\mathrm{p}} \mathrm{O}_{2} 99 \%$, Atemfrequenz etwa 50/Minute, ein exspiratorischer Stridor ist deutlich zu vernehmen, Pulsfrequenz laut Pulsoxymetrie 114/Minute - eine Blutdruckmessung sei bei dem aufgelösten, ängstlichen Kind noch nicht zu etablieren gewesen. Auch sei keine Ursache für das Geschehen zu erheben.

\section{Psychologische Aspekte}

Kindern ist es, in Abhängigkeit von ihrer Entwicklungsstufe, nicht immer möglich, die Situation zu erfassen, in der sie sich befinden. Daraus können situativ Verhaltensweisen entstehen, die den Zugang zum Kind erschweren oder gar unmöglich machen. Unter Umständen verzögert dies beispielsweise die Venenpunktion und eine notwendige Analgesie.

Unerwartetes Verhalten wie Weglaufen oder Wegziehen der Hand beim Anblick von Kanülen ist häufig, sodass Kinder niemals unbeobachtet sein sollten. Zur weiteren Verunsicherung kann beim Rettungsdienstpersonal und bei Bezugspersonen führen, dass Kinder sich nicht immer adäquat zu ihren Beschwerden äußern und je nach Entwicklungsstufe zum Fremdeln neigen.

\section{Ersteinschätzung}

Zur Ersteinschätzung empfehlen die American Heart Association und die American Academy of Pediatrics die Anwendung des pädiatrischen Untersuchungsdreiecks (PAT, Pediatric Assessment Triangle) [2]. Mithilfe des PAT können bereits binnen weniger Sekunden und ohne technisches Equipment strukturiert die folgenden Qualitäten erfasst werden:

- Aussehen/Verhalten

- Atmung

- Hautkolorit

Ergeben sich bei Anwendung des PAT Auffälligkeiten wie veränderte Atemfrequenz oder verändertes Hautkolorit, können bereits Rückschlüsse auf die Dringlichkeit der Situation, von Maßnahmen und ggf. auf die Ursache von Störungen (z. B. Zyanose und Apathie bei Bradypnoe) gezogen werden. Die Erfassung von Vitalparametern ist kein Bestandteil dieses Vorgehens; diese werden im weiteren Verlauf erhoben.

Neben dem strukturierten Vorgehen ist es von Vorteil, dass das Kind zur Ersteinschätzung oftmals in der körperlichen Nähe seiner Bezugsperson belassen werden kann. Dies erleichtert den Beziehungsaufbau und reduziert den Stress bei allen Beteiligten.

\section{FALLBEISPIEL}

Die Ersteinschätzung durch Notarzt und RTW-Besatzung erfolgt durch die Anwendung des PAT. Während sich Notarzt und Besatzung des RTW weiter dem Kind zuwenden, versucht der Notfallsanitäter des NEF bei der ebenfalls aufgeregten Großmutter eine Fremdanamnese zu erheben: Insbesondere in den Frühlingsmonaten leide die junge Patientin unter „Schnupfen und Kurzatmigkeit“, Medikamente nehme sie keine und sei sonst auch gesund.

\section{Allgemeine Verhaltenshinweise}

Noch mehr als bei Erwachsenen muss eine vertrauensvolle Atmosphäre für das Kind und -sofern vorhanden - für die Begleitperson geschaffen werden. Neben einer kindgerechten, ruhigen Ansprache sollte das Rettungsdienstpersonal sich dem Kind und seiner Bezugsperson ruhig nähern und diese mit in die Behandlung einbinden.

Beiden wird in angepasster Sprache alles erläutert, was geschieht oder geplant ist. Oftmals empfinden Eltern bzw. Bezugspersonen es als belastend, wenn sie ihrem erkrankten Kind nicht helfen können und keinen Zugang zu ihrem Kind haben [1]. Zudem können Eltern wichtige Hinweise für die Anamnese oder Informationen zu den körperlichen Gegebenheiten (Alter, Größe, Gewicht, Allergien) liefern.

Cave

Maßnahmen, die ein Kind nicht toleriert und die medizinisch nicht dringend geboten sind, sollten keinesfalls erzwungen werden.

Wann immer möglich, sollte den Eltern bzw. Bezugspersonen auch während der Versorgung der Zugang zum Kind ermöglicht werden - sofern darunter die Qualität der medizinischen Versorgung nicht leidet und das Rettungspersonal frei arbeiten kann.

\section{PRAXIS}

Tipp

Neben den Eltern empfiehlt es sich, abhängig von der Situation im Einsatz und den sozialen Verhältnissen, Großeltern, ältere Geschwister oder - je nach Entwicklungsstufe - auch Freunde des Kindes als Informationsquelle und als Bezugspersonen einzubinden. Neben dem reinen Informationsgewinn kann dies bei nicht vital bedrohlichen Notfällen zur Entspannung der Situation beitragen und den Beziehungsaufbau des Einsatzteams zum Kind erleichtern. 
Merke

Noch mehr als bei Erwachsenen gilt, dass sich Stress auf Kinder überträgt und den Einsatz erschwert.

\section{FALLBEISPIEL}

Den eingesetzten Kollegen gelingt es nach „Talkdown" und kindgerechtem Erläutern aller Maßnahmen, die Anamnese zu vervollständigen: Nach dem Trinken aus einer Getränkedose hatte die Patientin einen schmerzhaften Stich in der Zunge verspürt. Kurz darauf sei die Zunge angeschwollen und das Atmen erschwert gewesen.

\section{Anatomische Besonderheiten}

\section{Allgemeines}

Neben psychologischen Aspekten gibt es bei Kindern deutliche Unterschiede in der Anatomie. Diese müssen aufgrund der enormen Bedeutung für die Behandlung den Mitarbeitern im Rettungsdienst zumindest in groben Zügen bekannt sein.

\section{Größenverhältnisse}

Im Gegensatz zu Erwachsenen besitzen Kinder einen großen und im Verhältnis schwereren Kopf, der bei Dezelerationstraumata größeren Scherkräften ausgesetzt ist als bei Erwachsenen und damit ein erhöhtes Risiko für HWS-Verletzungen aufweist. Je nach Alter sind der Thorax und die Extremitäten noch klein, das Abdomen ist groß [3].

\section{Temperaturregulation}

Kinder haben eine im Vergleich zur Körpermasse große Körperoberfläche, sodass sie stärker hypothermiegefährdet sind [4]. Bei Kleinkindern und Säuglingen ist dies besonders ausgeprägt, sodass - auch bei normaler Umgebungstemperatur - besonderes Augenmerk auf den Wärmeerhalt gelegt werden muss.

\section{Zugang}

Da kleinere Kinder ein größeres subkutanes Fettgewebe aufweisen [5] und die Blutgefäße relativ klein sind, sind Venenzugänge oftmals schwer zu platzieren [1, 4], und der Versuch endet bei Ungeübten oftmals in Fehlpunktionen. Intuitive Abwehrbewegungen des Kindes tragen ein Übriges zum Misserfolg bei. Dies erhöht den Druck bei allen Beteiligten, sodass die Indikation besonders gut abgewogen werden sollte.
PRAXIS

Tipp

Als Alternative zum intravenösen hat sich insbesondere in den letzten Jahren der intraossäre Zugang bewährt, der auch von Ungeübten gut etabliert werden kann und als gleichwertig anzusehen ist [6]. Weite Verbreitung hat in den letzten Jahren z. B. die EZ- $1 \mathrm{O}^{\circledR}$ gefunden, die in Abhängigkeit des Patientenhabitus und -alters mit unterschiedlich langen Kanülen verwendet werden kann. Weitere Produkte (z. B. $\mathrm{Cook}^{\circledR}$-Nadel) sind hierzu ebenfalls kommerziell erhältlich und regional unterschiedlich vertreten.

\section{FALLBEISPIEL}

Bei vermutetem Insektenstich, der fremdanamnestisch zu eruierenden Allergieneigung und mittlerweile vervollständigten Vitalparametern ergibt sich folgende Arbeitsdiagnose:

Allergische Reaktion mit Schwellung der oberen Atemwege, Bronchokonstriktion, kreislaufstabil der Notarzt entschließt sich zur medikamentösen Therapie mit $\mathrm{H}_{1 / 2}$-Blockern, Prednisolon i.v. sowie Salbutamol über eine Inhalationsmaske.

\section{Der kindliche Atemweg}

Eine weitere Besonderheit stellt der kindliche Atemweg dar. Neben einer überproportional großen Zunge, die bei der Intubation den Blick auf die Stimmritze stören kann, besitzen Kinder einen langen U-förmigen Kehlkopf. Der Larynx steht höher und ventraler als beim Erwachsenen. Die engste Stelle befindet sich bis etwa zum 8.-10. Lebensjahr unter der Stimmritze [3, 5, 7]. Beim Erwachsenen befindet sie sich in Höhe des Ringknorpels. Für die Praxis bedeutet dies, dass bei der Intubation ein Tubus oder Führungsstab niemals mit Gewalt unter diese Höhe geschoben werden darf, da dies sonst zu erheblichen Verletzungen führen kann.

Ein Schleimhautödem infolge eines zu stark geblockten Cuffs muss unbedingt vermieden werden: Wegen des geringen Trachealdurchmessers führen bereits kleinere Schwellungen zu einer deutlichen Verengung der Trachea [3].

PRAXIS

Tipp

Bei der Lagerung zur Intubation empfiehlt es sich, wegen des großen Hinterkopfes des Kleinkindes bis ca. zum 2. Lebensjahr zwischen die Schulterblätter ein Tuch oder Ähnliches zu platzieren. Liegen der Hinterkopf und die Schultern auf einer ebenen Unterfläche auf, so kann es zu einem Verschluss der Atem- 
- Tab. 1 Physiologische Parameter [8].

\begin{tabular}{|c|l|l|l|}
\hline $\begin{array}{l}\text { Alter in } \\
\text { Jahren }\end{array}$ & $\begin{array}{l}\text { Atemfrequenz } \\
\text { (Atemzüge pro } \\
\text { Minute) }\end{array}$ & $\begin{array}{l}\text { Herzfrequenz } \\
\text { (Schläge pro } \\
\text { Minute) }\end{array}$ & $\begin{array}{l}\text { Blutdruck } \\
\text { (systolisch } \\
\text { in mm Hg) }\end{array}$ \\
\hline$<1$ & $30-40$ & $110-160$ & $70-90$ \\
\hline $2-5$ & $25-30$ & $95-140$ & $80-100$ \\
\hline $5-12$ & $20-25$ & $80-120$ & $90-110$ \\
\hline$>12$ & $15-20$ & $60-100$ & $100-120$ \\
\hline
\end{tabular}

wege kommen, und die Intubation wird durch eine Veränderung der optischen Achse erschwert [2].

\section{Physiologische Besonderheiten}

\section{Allgemeines}

Die physiologischen Gegebenheiten stellen Mitarbeiter des Rettungsdienstes mangels Routine oftmals vor Herausforderungen: Während Herzfrequenz, Blutdruck und Atemfrequenz wegen der vermeintlich geringen Bandbreite beim Erwachsenen routiniert eingeschätzt werden können, variieren diese bei Kindern in Abhängigkeit vom Alter sehr stark ( $\triangleright$ Tab. 1). Dies kann zu Fehleinschätzungen und damit zusätzlichem Stress im Team führen.

\section{Merke}

Je jünger das Kind, desto niedriger ist der systolische Blutdruck und desto höher sind Herz- und Atemfrequenz.

\section{Herz-Kreislauf-System und Atmung}

Während die Atemfrequenz aufgrund eines relativ erhöhten Stoffwechsels und damit einer erhöhten $\mathrm{CO}_{2}$-Produktion sowie eines erhöhten $\mathrm{O}_{2}$-Bedarfs bei gleichzeitig kleinen Lungenvolumina erhöht ist, beruht die erhöhte Herzfrequenz auf dem niedrigen systemischen Gefäßwiderstand. Dieser nimmt erst mit dem Alter zu. Das Herzminutenvolumen ist gerade bei jüngeren Kindern hauptsächlich von der Herzfrequenz abhängig. Dies erklärt, warum eine für Erwachsene normwertige Herzfrequenz für Säuglinge als lebensbedrohlich zu werten ist! Zugleich reagiert das kindliche Herz empfindlicher auf Hypoxie, was schneller als beim Erwachsenen zur Bradykardie führt.

Soll ein Kind notfallmäßig narkotisiert und intubiert werden, wird vor diesem Hintergrund oft zu einer modifizierten schnellen Narkoseeinleitung geraten. Statt der üblichen Abfolge (Gabe der Narkosemedikamente, keine Zwischenbeatmung, Intubation) kann das Kind mit möglichst geringen Spitzenbeatmungsdrücken zwischen der Medikamentengabe und der Intubation zwischenbeat- met werden. Damit wird der Sicherheitsfaktor für das Kind erhöht.

\section{Blut, Volumen und medikamentöse Besonderheiten}

Das Blutvolumen ist beim Kind im Vergleich zum Erwachsenen pro Kilogramm Körpergewicht zwar erhöht (rund $80 \mathrm{ml} / \mathrm{kg} \mathrm{KG}$ ) [3], das Gesamtvolumen (in Litern) ist aber wesentlich geringer. Somit führt der Verlust kleinerer Blutmengen im Vergleich zum Erwachsenen prozentual gesehen zu einem höheren Blutverlust.

\section{Blutverluste werden bei Kindern oft unterschätzt! Kinder kompensieren Volumendefizite, lange bevor die Situation rasch dekompensiert.}

Bezogen auf die Gesamtmasse des Körpers haben Kinder zudem einen höheren Wasseranteil, der sich erst im Laufe des Wachstums dem des Erwachsenen angleicht. Dies hat praktische Konsequenzen für den Alltag: Kinder dehydrieren bei Infekten und höheren Temperaturen schneller [4]. Allerdings muss die Flüssigkeitszufuhr bei Infusionen im Auge behalten werden: Für Erwachsene übliche Volumenmengen können beim Kind schnell zu einer Überwässerung führen [4].

Bewährt hat sich eine an das Körpergewicht angepasste statt der blinden Flüssigkeitsgabe. Zudem müssen Medikamente u. a. wegen anderer Verteilungsvolumina, veränderten Abbaus und anderer Ausscheidung differenziert dosiert werden [5] bzw. sind im Kindesalter nicht zugelassen.

\section{FALLBEISPIEL}

Während der Notarzt auf seinem Smartphone nach der geeigneten Dosierung für die Patientin sucht, holt die Rettungssanitäterin eine Kinderinhalationsmaske aus dem RTW und bereitet anschließend den Transport in die Kinderklinik des Nachbarorts vor.

\section{Hilfsmittel und logistische Besonderheiten}

\section{Equipment zur Sicherung der Atemwege und weitere Hilfsmittel}

Die Mindestausstattung für die Notfallausrüstung legt die DIN 13232 fest. Neben Basisausstattung und Material für die Versorgung Erwachsener beschreibt der Teil C die Materialvorhaltung für Kinder und Säuglinge. Das Material kann modular in verschiedenen Koffer- oder Rucksacksystemen oder kombiniert mitgeführt werden. Es bietet 
sich jedoch an, Equipment für den Kindernotfall separat vorzuhalten.

Aufgrund regional unterschiedlicher Ausstattungsstandards kann das vorgehaltene Material in Umfang, Qualität und Verlastung variieren [9]. Um Zeitverluste bei der Durchführung lebensrettender Maßnahmen zu verhindern, müssen die jeweils eingesetzten Materialen und ihre Verlastung in Rucksack oder Koffer dem Rettungsfachpersonal bekannt sein.

Hilfsmittel zur Sicherung der Vitalfunktionen sind prinzipiell denen erwachsener Patienten gleich. Jedoch sind sie naturgemäß kleiner und variieren nach den altersbedingten anatomischen Gegebenheiten. Die richtige Größenauswahl kann das Rettungsdienstpersonal vor Probleme stellen, da oftmals die Routine fehlt.

Unter den Beatmungsmasken sollen möglichst solche mit geringem Totraumvolumen genutzt werden. Um bei den geringen Lungenvolumina eine Verabreichung zu hoher Tidalvolumina zu vermeiden, bieten sich spezielle Kinderbeutel mit kleinerem Volumen an. Die Atemfrequenz muss altersadaptiert sein und kann beim Säugling bei bis zu 70/min liegen [1].

\section{Cave}

Werden Beatmungsgeräte genutzt, muss besonderes Augenmerk auf den einstellbaren Atemzugvolumina liegen. Bei manchen Respiratoren kann im Kindernotfallmodus das Atemzugvolumen unbegrenzt gesteigert werden!

\section{Endotracheale Intubation}

Lässt sich eine Intubation nicht vermeiden, gilt als Faustregel bei Endotrachealtuben, dass die benötigte Größe mit dem Durchmesser des Kleinfingers des Kindes korreliert. Alternativ bietet die folgende Gleichung Hinweise auf den Innendurchmesser des Tubus [7]:

$4+\frac{\text { Alter }}{4}=$ Innendurchmesser des Tubus in Millimeter

Das Bereitlegen je eines nächstgrößeren bzw. kleineren Durchmessers hat sich angesichts der individuellen anatomischen Unterschiede bewährt.

Zur Vermeidung bronchialer Intubationen muss eine dem Alter angepasste Intubationstiefe beachtet werden. Da insbesondere im 1. Lebensjahr die anatomischen Gegebenheiten noch deutlich vom Kleinkind differieren können, wird zur Intubation ein gerader Spatel empfohlen, der dieser Tatsache Rechnung trägt [3].
FALLBEISPIEL

Bewertende Stellungnahme

In der Fallschilderung stand zunächst die Feststellung einer möglichen vitalen Bedrohung im Vordergrund. Nach Stellen der Arbeitsdiagnose liegen nun weitere Stolpersteine vor dem eingesetzten Personal: Da Einsätze mit Kindern selten sind, die speziellen Anforderungen der verschiedenen Altersgruppen variieren und es wenig Routine mit dem vorgehaltenen Equipment gibt, ist besondere Aufmerksamkeit gefragt.

Medikamentengabe

Cave

Dosierungsfehler sind bei Kindern häufig. Schätzfehler beim Körpergewicht, Verdünnungs- und Rechenfehler können gravierende Folgen haben [10].

Der Einsatz von Hilfsmitteln ist daher nicht nur für den pädiatrisch unerfahrenen Anwender sinnvoll und kann Dosierungsfehler vermeiden. Taschenrechner, Apps mit voreingestellten Medikamenten oder im Notfallkoffer bereitliegende Tabellen sind mögliche Beispiele.

Ein bekanntes Hilfsmittel ist das Pädiatrische Notfalllineal ${ }^{\circledR}$. Das Lineal wird neben das zu versorgende Kind gelegt und die Körpergröße abgelesen. Da die Körpergröße für einen Altersbereich typisch ist, werden dem mutmaßlichen Alter entsprechende physiologische Normalwerte, Standardgrößen für Material wie Tuben oder Laryngoskopiespatel sowie Dosierungen der wichtigsten Medikamente - basierend auf dem Idealgewicht - zugeordnet.

Schätzungen des Gewichts ebenso wie das Zugrundelegen des tatsächlichen Gewichts können insbesondere bei adipösen Kindern zur Überschätzung des Verteilungsvolumens der Medikamente und damit zu Überdosierungen führen [11].

Anhaltspunkte zur Einschätzung des Alters und des Körpergewichts gibt $\downarrow$ Tab. 2 im Überblick.

Merke

Das Verwenden dem Personal bekannter Hilfsmittel

kann das Risiko von Dosierungsfehlern verringern und Handlungssicherheit schaffen.

\section{Transport}

Ist eine klinische Weiterversorgung notwendig, wird idealerweise eine Einrichtung mit pädiatrischer Abteilung ausgewählt. Steht diese nicht zeitnah zur Verfügung, müssen in Zuweisungskonzepten im jeweiligen Rettungs- 
- Tab. 2 Einschätzung des Alters pädiatrischer Patienten.

\begin{tabular}{|l|l|l|}
\hline Merkmal & Alter ca. & Gewicht $\mathbf{( k g )} \mathbf{c a}$. \\
\hline Keine Zähne & 6 -8 Monate & 6 \\
\hline Sitzt frei & 8 Monate & 8 \\
\hline Steht mit Hilfe & 10 Monate & 9 \\
\hline Läuft ohne Hilfe & 15 Monate & 10 \\
\hline Einzelne Wörter & 18 Monate & 12 \\
\hline Zwei-Wort-Sätze & 2 Jahre & 13 \\
\hline Motorik koordiniert & 3 Jahre & 15 \\
\hline Zahnlücken & 6 - 7 Jahre & 22 \\
\hline
\end{tabular}

dienstbereich alternative Versorgungseinrichtungen benannt sein.

Beim abschließenden Transport gilt es die Besonderheiten im Kindesalter zu beachten: Die geringere Körpergröße macht spezielle Sicherungsmaßnahmen obligat. Sind keine Kinderrückhaltesysteme in den Tragen verbaut, stehen Nachrüstsysteme für Tragesysteme aller gängigen Hersteller zur Verfügung. Deren Anwendung und die jeweiligen Grenzen für Körpergröße und Gewicht müssen dem Personal bekannt sein.

Für kleinste Patienten bieten auch Kindersicherungsschalen der Eltern Transportsicherheit, sofern die Platzverhältnisse eine adäquate Sicherung des Systems im RTW zulassen. Der Transport kleiner Patienten ohne eigenen Sitzplatz mit Sicherungssystem oder -gurt auf dem Schoß der Eltern ist selbst angesichts des Arguments der vermeintlichen Beruhigung des Kindes in jedem Fall abzulehnen [12]. Es obliegt dem Fahrer des Rettungsmittels zu überprüfen, ob bei Fahrtantritt alle Insassen gesichert sind.

PRAXIS

Prinzip

Jeder Insasse im Rettungsmittel muss zwingend auf einem Sitz- oder Liegeplatz mit einem Rückhaltesystem gesichert sein. Bei Kindern muss dieses zu Körpergröße und Gewicht passen.

\section{Teammanagement}

Die Kommunikation im Team hat nicht nur beim Kindernotfall einen hohen Stellenwert. Die Nutzung aller Ressourcen und die Einbeziehung von Meinungen aus dem Team können den Einsatzverlauf positiv bestimmen. Klare Ansagen und die Bestätigung des Empfängers im
Ping-Pong-Prinzip („Bereite bitte 2 mg Fenistil vor“ „Ich habe 2 mg Fenistil aufgezogen“) vermeiden Unklarheiten und reduzieren Fehler.

\section{PRAXIS}

Tipp

Insbesondere bei ungewohnten Medikamentendosierungen bietet das Wiederholen der Dosierungen einen Sicherheitspuffer: Alle Teammitglieder hören die Anforderungen ein zweites Mal und können bei als falsch empfundenen Dosierungen Bedenken äußern.

Kindereinsätze werden häufig als Belastung wahrgenommen [13]. Die Nachbesprechung im Team, insbesondere nach ungünstigem Verlauf, hilft bei der Verarbeitung von Eindrücken. Gemeinsam können Fehlerquellen konstruktiv aufgearbeitet und auch positive Abläufe besprochen werden und so eine größere Routine für zukünftige Einsätze schaffen.

\section{Fazit}

Kindernotfälle erfordern ein spezielles Vorgehen. Da sie im Alltag selten sind, empfiehlt sich eine Vorbereitung durch entsprechendes Training wie z. B. den Besuch auf Kindernotfälle ausgerichteter Kurse (z. B. PALS ${ }^{\circledR}$ ) und Fortbildungen. Dies erhöht die Sicherheit und reduziert auftretende psychische Belastungsfaktoren bei allen Beteiligten.

\section{KERNAUSSAGEN}

- Kindernotfälle sind selten und erfordern ein strukturiertes, altersabhängiges Vorgehen unter Beachtung des jeweiligen Entwicklungsstandes des Kindes.

- Die Teilnahme an speziellen Kursformaten kann die Sicherheit beim Personal im Umgang mit Kindernotfällen erhöhen und damit Stress bei allen verringern.

- Eltern bzw. Bezugspersonen sollten wann immer möglich mit in die Versorgung eingebunden und betreut werden, da dies auf alle Anwesenden beruhigend und stabilisierend wirkt.

- Die Kenntnis physiologischer Besonderheiten ist essenziell für eine adäquate Notfallversorgung.

- Unerfahrene sollten Hilfsmittel nutzen, um physiologische Parameter, Medikamentendosierungen oder die Größe benötigter Materialien einschätzen zu können. 
- Gelebte Teamarbeit und gleichberechtigte Kommunikation ermöglichen es, Fehlerquellen aufzudecken und anzusprechen.

- Konstruktive Nachbesprechungen im Team helfen, auch kritische Einsätze zu verarbeiten und gemeinsam Routine für folgende Notfallsituation mit Kindern zu festigen.

\section{Interessenkonflikt}

Die Autoren geben an, dass kein Interessenkonflikt besteht.

\section{Autorinnen/Autoren}

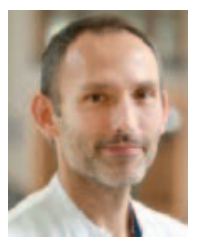

\section{Thomas Rielage}

Jahrgang 1978. 2002-2008 Studium der Humanmedizin an der Westfälischen WilhelmsUniversität Münster. 2008-2014 Facharztausbildung Anästhesiologie. 2014 Facharzt für Anästhesiologie. Zusatzweiterbildungen: Notfallmedizin 2011, Qualitätsmanagement 2012, Palliativmedizin 2015, Suchtmedizin 2018, 2014-2018 Leitender Notarzt bei der Stadt Dortmund. Seit 8/2018 Oberarzt Anästhesiologie am Klinikum Osnabrück. Schwerpunkte: Notfallmedizin, Ausbildung, u. a. Paediatric Advanced Life Support ${ }^{\circledR}$.

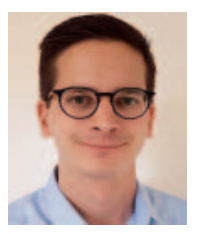

\section{Fabian Biederbick}

Jahrgang 1988. Seit 2008 im Rettungsdienst, Rettungsassistent mit Zusatzqualifikationen u. a. als Organisatorischer Leiter Rettungsdienst und im Intensivtransport. 2010-2017 Studium der Humanmedizin an der Ruhr-Universität Bochum. Seit 2018 Facharztweiterbildung Anästhesiologie am Alfried Krupp Krankenhaus Essen.

\section{Korrespondenzadresse}

\section{Thomas Rielage}

Klinikum Osnabrück

Klinik für Anästhesiologie und operative Intensivmedizin

Am Finkenhügel 1

49076 Osnabrück

thomasrielage@web.de
[1] Gerdts KG. Pädiatrische Notfälle. In: Kühn D, Luxem J, Runggaldier K, Hrsg. Rettungsdienst heute. 5. Aufl. München: Elsevier, Urban \& Fischer Verlag; 2010: 523-526

[2] American Heart Association (AHA). PALS - Erweiterte Maßnahmen der Reanimation bei Kindern. Mesquite, Texas: American Heart Association; 2017

[3] Jöhr M (Hrsg) Kinderanästhesie. 8. Aufl. München: Elsevier, Urban \& Fischer Verlag; 2013

[4] Ziegenfuß T (Hrsg) Notfallmedizin. 7. Aufl. Heidelberg: Springer Verlag; 2017: 387. doi:10.1007/978-3-662-52775-7_15

[5] Striebel HW (Hrsg) Die Anästhesie. Band II. 2. Aufl. Stuttgart: Schattauer; 2010

[6] Deutsche Gesellschaft für Anästhesiologie und Intensivmedizin e. V. (DGAl). Die intraossäre Infusion in der Notfallmedizin (31.10.2017). Im Internet: https://www.awmf.org/uploads/ tx_szleitlinien/001-042I_S1_Der-intraossaere-Gefaesszu gang-in-der-Notfallmedizin_2018-02.pdf; Stand: 12.12.2018

[7] Campbell JE (Hrsg) Präklinische Traumatologie. 6. Aufl. München: Pearson Studium; 2010: 305

[8] Wiese S. Pädiatrische Notfälle. In: Brokmann J, Rossaint R, (Hrsg) Repetitorium Notfallmedizin. 2. Aufl. Heidelberg: Springer Verlag; 2012: $281-282$

[9] Thöns M, Sefrin P. Vorhaltung notfallmedizinischen Equipments für den Kindernotfall. Der Notarzt 2007; 23: 117-122

[10] Kaufmann J, Laschat M, Wappler F. Medication errors in pediatric emergencies - a systematic analysis. Dtsch Aerztebl Int 2012; 109: 609-616

[11] Kaufmann J, Roth B, Engelhardt T et al. Development and Prospective Federal State- Wide evaluation of a device for height-based dose recommendations in prehospital pediatric emergencies: a simple tool to prevent most severe drug errors. Prehospital Emergency Care 2018; 22: 252-259

[12] Bundesministerium der Justiz. Straßenverkehrs-Ordnung. § 21 Personenbeförderung (August 2018). Im Internet: https:// www.gesetze-im-internet.de/stvo_2013/__21.html

[13] Karutz H. Psychische Belastungen von Helfern bei Einsätzen mit Kindern. In: Karutz H, Juen B, Kratzer D, et al., (Hrsg) Kinder in Krisen und Katastrophen. Spezielle Aspekte psychosozialer Notfallversorgung. 1. Aufl. Innsbruck: Studia Universitätsverlag; 2017: 531-539

Bibliografie

DOI https://doi.org/10.1055/a-0647-9945

retten 2019; 8: 23-31

(c) Georg Thieme Verlag KG, Stuttgart · New York

ISSN 2193-2387 
Punkte sammeln auf CMEthieme.de

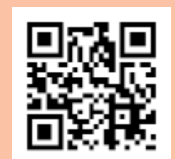

Diese Fortbildungseinheit ist in der Regel 12 Monate online für die Teilnahme verfügbar.

Den genauen Einsendeschluss finden Sie under https://eref.thieme.de/CXB4WIQ.

Sollten Sie Fragen zur Online-Teilnahme haben, finden Sie unter https://cme.thieme.de/hilfe

eine ausführliche Anleitung. Wir wünschen viel Erfolg beim Beantworten

der Fragen!

Unter https://eref.thieme.de/CXB4WIQ oder über den QR-Code kommen Sie

direkt zur Startseite des Wissenstests.

\section{Frage 1}

Welche Aussage zur Einbindung von Bezugspersonen im Kindesalter ist richtig?

A Sie können durch emotionales Verhalten die Arbeit des Rettungsdienstes behindern und können daher nicht in die Behandlung eingebunden werden.

B Sie sollten wegen der psychischen Belastung grundsätzlich zum Verlassen der Einsatzstelle aufgefordert werden.

C Bezugspersonen und Kinder sind in der Regel bei Notfällen dermaßen überfordert, dass Erklärungen zum Vorgehen und den gewählten Maßnahmen überflüssig sind.

D Sie sollten, wann immer möglich, mit in die Behandlung eingebunden werden, um den Stress bei allen Beteiligten zu reduzieren.

E Unter Bezugspersonen sind nur die Erziehungsberechtigten zu verstehen.

\section{Frage 2}

Welche Aussage zum kindlichen Atemweg trifft zu?

A Die Epiglottis ist U-förmig und steht beim Kind niedriger als beim Erwachsenen.

B Der Blick auf die Stimmritze wird bei der Intubation durch die kleine Zunge des Kindes erleichtert.

C Da Kleinkinder einen relativ großen Hinterkopf besitzen, sollte man diesen zur Erleichterung bei der Intubation unterpolstern. Dadurch verbessert sich die optische Achse.

D Kleinere Schwellungen in der Trachea sind für das Kind immer unproblematisch.

E Die engste Stelle in der Trachea bei Kindern bis ca. zum Ende der Grundschule befindet sich subglottisch.

\section{Frage 3}

Nur eine der folgenden Aussagen zur Dosierung von Medikamenten bei Kindernotfällen ist zutreffend. Welche?

A Es gilt für alle Medikamente, dass sie sicher mit halber Erwachsenendosis bei Kindern angewandt werden können.

B Medikamentenfehldosierungen treten nur bei Unerfahrenen auf und sind somit durch Training gerade in Notfallsituationen immer zu vermeiden.

C Medikamentenfehldosierungen treten bei Kindernotfällen häufig und auch bei Erfahrenen auf. Die Anwendung eines Hilfsmittels wie des Pädiatrischen Notfalllineals, bei dem pro Kilogramm Körpergewicht ausgerechnete Dosierungen abgelesen werden können, verringert das Risiko.

D Entgegen einer verbreiteten Annahme gibt es keine physiologischen Besonderheiten bei Kindern beim Abbau, der Ausscheidung und der Umverteilung von Medikamenten im Vergleich zu Erwachsenen.

E Auch bei Kindern dürfen alle Medikamente bedenkenlos genutzt werden, die beim Erwachsenen zugelassen sind.

\section{Frage 4}

Welche Aussage zu Besonderheiten bei Kindern trifft zu?

A Die Körperoberfläche ist bei Kindern im Vergleich zur Körpermasse kleiner als bei Erwachsenen, weshalb sie zum Auskühlen neigen.

B Der Körper ist im Vergleich zum Kopf größer, weshalb Kinder bei Dezelerationstraumata kein größeres Risiko für ein Schleudertrauma haben als Erwachsene.

C Herzgesunde Kinder sind bei Sauerstoffmangelversorgung nicht so gefährdet, eine Bradykardie zu entwickeln wie Erwachsene.

D Kinder besitzen einen relativ großen Kopf und Hinterkopf im Vergleich zum Körper; bei Dezelerationstraumata besitzen sie damit ein erhöhtes Risiko für HWS-Verletzungen.

E Das Blutvolumen beträgt beim Kind $50 \mathrm{ml} / \mathrm{kg}$ Körpergewicht und ist damit größer als beim Erwachsenen.

- Weitere Fragen auf der folgenden Seite... 


\section{Punkte sammeln auf CME.thieme.de}

\section{Frage 5}

Wodurch ist die Physiologie beim Kind gekennzeichnet?

A Atem- und Herzfrequenz sind bei Kindern über alle Altersgruppen hinweg nahezu konstant und ändern sich erst zur Pubertät hin in Richtung der Normwerte von Erwachsenen.

B Da bei Kindern der Gefäßwidertand erst mit fortschreitendem Alter zunimmt, regulieren Kinder ihr Herzzeitvolumen hauptsächlich über die Herzfrequenz.

C Kleinere Blutverluste sind bei Kindern immer harmlos, da sie pro Kilogramm Körpergewicht mehr Blut besitzen als Erwachsene.

D Aufgrund der geringeren Körpergröße und des geringeren Körpergewichts von Kindern sind der Stoffwechsel und der Sauerstoffbedarf erniedrigt.

E Kleinere Kinder können Flüssigkeitsverluste besser kompensieren als Erwachsene.

\section{Frage 6}

Nur eine der folgenden Aussagen zur endotrachealen Intubation trifft zu. Welche?

A Gerade bei kleineren Kindern gibt es keine Möglichkeit, die Tubusgröße vorab einzuschätzen.

B Im Zweifel ist der Tubus zu wählen, dessen Durchmesser dem des Kleinfingers des Notarztes entspricht.

C Der Innendurchmesser des zu wählenden Tubus lässt sich mit der Formel (Alter des Kindes/4)+ 4 abschätzen.

D Es sind immer Tuben mit Cuff zu wählen und zur Aspirationsprophylaxe eher großzügig zu blocken.

E Insbesondere bei kleinen Kindern sind zwingend gebogene Spatel zu verwenden, um die bestmögliche Sicht zu schaffen.

\section{Frage 7}

Wodurch ist das Auftreten von Kindernotfällen im Rettungsdienst gekennzeichnet?

A Der Anteil der Kindernotfälle ist mit rund $4 \%$ bereichsübergreifend klein.

B Kindernotfälle sind selten lebensbedrohlich und müssen daher nie unter Inanspruchnahme von Sonder- und Wegerechten angefahren werden.

C Die kardiopulmonale Reanimation oder die invasive Sicherung des Atemwegs sind regelmäßig notwendige Maßnahmen bei Kindern.

D Bradykardie und Kreislaufstilland haben meist eine kardiologische Ursache.

E Bei traumatologischen Notfällen treten HWS- und Kopfverletzungen aufgrund der kindlichen Proportionen seltener auf als bei Erwachsenen.

\section{Frage 8}

Eine der folgenden Aussagen bezüglich der Kommunikation am Einsatzort trifft nicht zu. Welche?

A Alle Maßnahmen sollten ohne weitere Erklärung durchgeführt werden, um das Kind nicht zu verunsichern.

B Das eingesetzte Personal sollte gleichberechtigt miteinander kommunizieren, um Ideen und Erfahrungen aus dem Team nutzen zu können.

C Medikamentendosierungen sollten im „Ping-Pong-Prinzip“ wiederholt werden, um Dosierungsfehler zu vermeiden.

D Den Kindern und ihren Bezugspersonen sollten alle Arbeitsschritte erläutert und, wenn es die Zeit erlaubt, Maßnahmen z. B. auch an Eltern oder Kuscheltieren demonstriert werden.

E Eine konstruktive Nachbesprechung im Team sollte stattfinden, insbesondere nach hektischen Einsätzen oder wenn diese von Beteiligten als negativ erlebt wurden.

\section{Frage 9}

Wodurch ist die Materialausstattung für pädiatrische Notfälle auf dem Rettungsmittel gekennzeichnet?

A Die Ausstattung mit Notfallmedikamenten ist bundeseinheitlich vorgegeben und darf nicht verändert werden.

B Material für die Versorgung von Kindern und Neugeborenen ist immer separat vorzuhalten, um Verwechslungen zu vermeiden.

C Normen regeln Mindestausstattungen für Rettungsmittel und Notfallkoffersysteme, diese sollten aber an die regionalen Standards und Verfahrensanweisungen angepasst und gegebenenfalls aufgerüstet werden.

D Für Kinder muss ein separates Beatmungsgerät bereitstehen.

E Da Kindernotfälle selten sind, muss über den Inhalt des Kindernotfallkoffers keine Kenntnis vorliegen.

\section{Frage 10}

Nur eine der Aussagen zum Transport trifft zu. Welche?

A Bei der Wahl der Klinik brauchen keine Besonderheiten beachtet zu werden.

B Befindet sich ein Elternteil im Fahrzeug, so ist zur Beruhigung die Mitnahme des Kindes auf dessen Schoß wünschenswert.

C Eltern sollten auf keinen Fall im Rettungsmittel das Kind begleiten, da sonst immer der Behandlungsablauf gestört wird.

D Es stehen integrierte und nachrüstbare Transportsicherungssysteme zur Verfügung, auf denen Kinder bis zum Neugeborenenalter adäquat gesichert werden können.

E Die Verwendung von Kinderrückhaltesystemen der Eltern ist nicht zulässig, da eine Einweisungspflicht für alle im Rettungseinsatz verwendeten Materialien besteht. 